\title{
A SINGLE-MASK PROCESS FOR MICROMACHINED MAGNETIC DEVICES
}

\author{
Florent Cros, Kieun Kim, and Mark G. Allen \\ School of Electrical and Computer Engineering \\ Georgia Institute of Technology \\ Atlanta, GA 30332-0250
}

\begin{abstract}
A method of fabrication is presented in which interlinked, electrically isolated magnetic coils and cores can be realized using a self-aligned, single-mask process. The process relies on two electroplating techniques: the differential filling and subsequent removal of metals in different aspect-ratio trenches of the same mold, in combination with more conventional plate-through-mold techniques. Since both the electromagnetic coil and the magnetic core are fabricated using the same mold, only one photolithographic process is required. Different materials can be deposited into the different aspect ratio portions of the mold. For example, in the fabrication of magnetic actuators, copper is used to fill in the high aspect-ratio winding trenches while $\mathrm{NiFe}$ permalloy is used to fill in the low aspect-ratio core trenches. Since the process results in electrically-self-isolated structures, the molds do not need to be removed and subsequent planarization issues (e.g., for surface micromachined devices) are greatly ameliorated. Actual geometrical limits (differences in aspect ratio) for which the process still yields functional devices were also investigated. Utilizing this process, functional electromagnetic coils of coil line widths of 15 and $50 \mu \mathrm{m}$ have been fabricated and tested.
\end{abstract}

\section{INTRODUCTION}

Micromachined magnetic components, sensors, and actuators have application in many areas, including integrated passive components [1], magnetic field sensing [2], large-stroke actuation [3], and electrical power generation on the microscale [4]. One major drawback of magnetic microdevices, however, is the relatively complex fabrication procedure required to interlink and isolate conductors and core materials. In addition, the relatively large thickness $(10-100 \mu \mathrm{m})$ of coils and cores required for efficient operation of these devices could present substantial planarization challenges.

As an example, consider the process developed by Taylor et al. [3] for the fabrication of magnetically-actuated, surfacemicromachined relays. This process is based on conventional plate-through-mold techniques [5]. In the fabrication of the electromagnetic driving element for this relay, three masks are required: one to define lower permalloy magnetic cores, one to define copper conductors, and one to define magnetic side cores. In addition, alignment between the copper conductors and the magnetic cores is critical to prevent electrical shorting. Since thick metal lines for coil and core are required, these alignment tolerances must be maintained through thick polymer molds. The extra space required for these tolerances limits the winding packing efficiency. Moreover, in order to benefit from 'taller' (i.e., larger cross-sectional area and high aspect ratio) conductors, while simultaneously leaving the upper surface reasonably planar for subsequent magnetic via etching and surface micromachining steps, difficult replanarization issues must be addressed. A poor degree of planarity may, for example, jeopardize subsequent processing steps such as the critical dry etch through the thick polymer molds in order to define wells for the magnetic side cores.

In this work, a method that avoids many of the above issues is presented. This process is based on conformal mold-filling techniques. Although conformal mold-filling techniques for other materials, e.g. metals [6] and silicon/polysilicon [7,8] have been previously developed, this approach differs from the previous approaches in that the deposited material is not removed by an anisotropic removal step such as chemical-mechanical polishing or reactive-ion etching. Instead, an isotropic wet etch and the differences in aspect ratios are utilized to selectively and completely (e.g., from sidewalls as well as the bottoms of trenches) remove the deposited metal from the lowest aspect ratio trenches. In addition, the mold can remain as an integral part of the final structure. This technique can also be extended (e.g., for magnetic devices) by combining it with a conventional plate though mold process. At the conclusion of this process, both the conductor and the ferromagnetic material are micromachined out of a single mold and remain embedded in the mold. Since it is not necessary to remove the molds to isolate the cores and windings, replanarization of thick electroplated structures is not required. In addition, the lack of necessity of mold removal makes the use of hard-toremove, but otherwise desirable, materials (such as SU-8 epoxy) very attractive. These two advantages greatly simplify the fabrication process, potentially removing many of the obstacles to commercially viable magnetic component fabrication.

\section{PROCESS}

A schematic of the single-mask fabrication process is described in Figure 1. This process requires the use of a robust material able to yield molds with both high and low aspect ratio trenches. A variety of polymer micromolding techniques, such as conventional photoresists, dry-etched polyimide, and thick, negative tone epoxy resists are suitable candidates. For example, conventional thick photoresists such as AZ 9260 allow structuring of thick mold layers and offer simultaneous high and low aspect ratio molds [9]. However, the major advantage of these materials in conventional plating approaches, i.e. that they are relatively easy to remove, is not required by this process (and in fact their relatively poor stability compared with epoxy-based resists can be a drawback in this process). Thus, in this work, SU-8 epoxy photoresist is used to fabricate the mold. As demonstrated in the literature, this polymer can yield very high aspect ratio trenches with nearly vertical side-walls [10-12]. 
A detalled process example is given below. A $1000 \mu \mathrm{m}$ thick NiFe (permalloy) substrate wafer is coated with a thin $(5 \mu \mathrm{m})$ layer of SU-8 epoxy photoresist to insulate the magnetic substrate. The layer is pre-baked, cross-linked and post-baked [10], in order to create a uniform isolation layer. On top of this layer, a thicker SU-8 layer is deposited and lithographically patterned to create a micro-mold (Fig 1.1). A key characteristic of this micromold is that the sections which will ultimately define windings are of a higher aspect-ratio (thickness:width) than the sections which will ultimately define the magnetic cores. The mold is conformally coated with a seed layer of Ti/Cu/Ti $(100 \mathrm{~nm} / 1000 \mathrm{~nm} / 100 \mathrm{~nm})$ using DC sputtering (Fig 1.2). Copper is then electroplated over the entire wafer, and the high aspect-ratio molds are filled first (Fig $1.3,1.4)$. Once the high aspect ratio molds are filled, the electrodeposited $\mathrm{Cu}$ layer is etched back (Fig 1.5). A variety of recipes were used in order to etch back the $\mathrm{Cu}$ (Fig 1.5); the SU-8 mold proved to withstand both acidic and basic etchants.

Note that the copper that coats any surface other than in the high aspect-ratio trenches, including that copper in the core areas, is much thinner than that in the high aspect-ratio molds. As a result, the copper (and seed layer) is selectively removed from lower aspect ratio molds as well as any flat opened surface in the field of the micromold, without the need for a lithography step (Fig 1.5). An additional benefit is that the copper lines are thereby electrically self-isolated. The insulation layer in the low aspect ratio areas is then removed in a self-aligned blanket dry etch down to the metallic surface of the substrate (Fig 1.6). Note that because the insulation layer is much thinner than the polymer mold, the blanket dry etch step does not significantly affect the height of the mold. Using the substrate as an electrical contact, NiFe is selectively electroplated, from the bottom of the NiFe substrate, filling the core mold (Fig 1.7). When the NiFe electroplating is complete, the surface has become reasonably self-planarized.

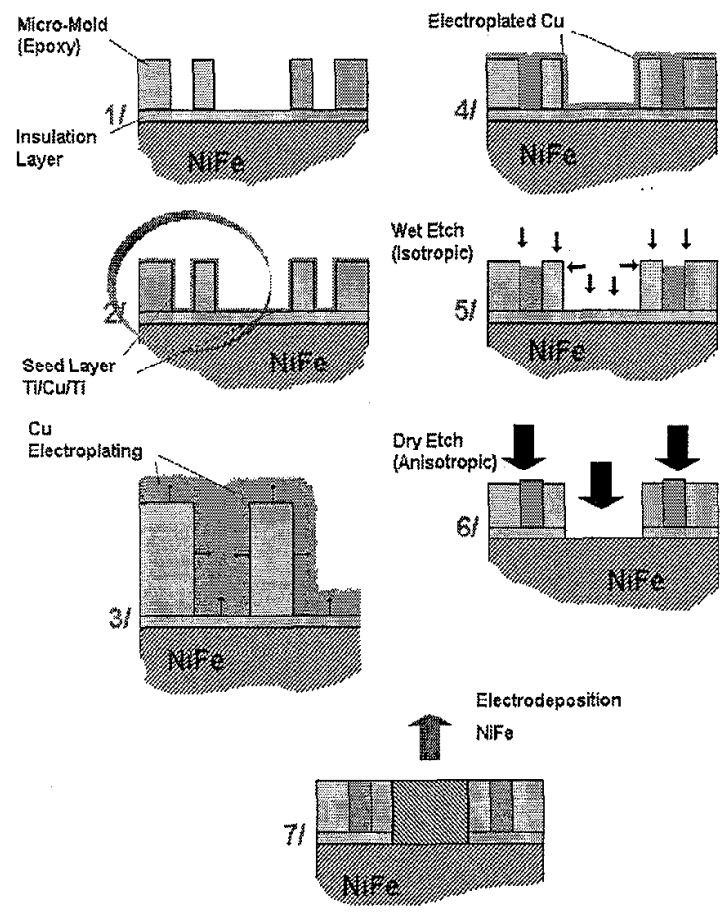

Figure 1: Schematic of the single mask process

\section{DISCUSSION}

In order to generate a high magnetic force yet maintain a relatively low driving current in a micromachined magnetic device, it is necessary to increase the number of turns in the coil (thereby increasing the number of ampere-turns in the magnetic circuit). At the same time, in order to make efficient use of the real-estate available, the highest possible packing density for the coil structure is desirable. Therefore, it is of interest to determine the actual geometrical limits (difference between low and high aspect ratio) for which the process still yields functional devices. The limit depends on the successful removal of the deposited metal from the lower aspect ratio trench while keeping the metal in the higher aspect ratio trench. Several test structures were created to probe these limitations. A $50 \mu \mathrm{m}$ thick epoxy mold with height-to-width aspect ratio trenches ranging from 5 to 0.25 were fabricated, and subjected to steps 1-5 of Figure 1. The etch back was stopped when it was observed that the copper within the highest aspect ratio trenches began to be removed. $\mathrm{Cu}$ was successfully completely removed from trenches as narrow as 60 microns (aspect ratio close to unity) while higher aspect ratio trenches are still filled with copper. Figure 2.a and 2.b illustrates the differential filling and removal of copper from different aspect-ratio trenches.

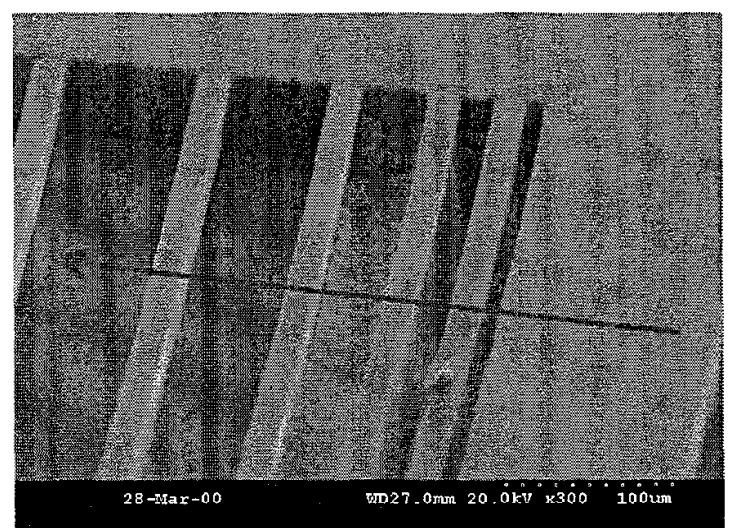

Figure 2.a: SEM photomicrograph of different aspect-ratio trenches. Notice that the wider trenches are devoid of metal whereas the narrower ones are still partially filled with them.

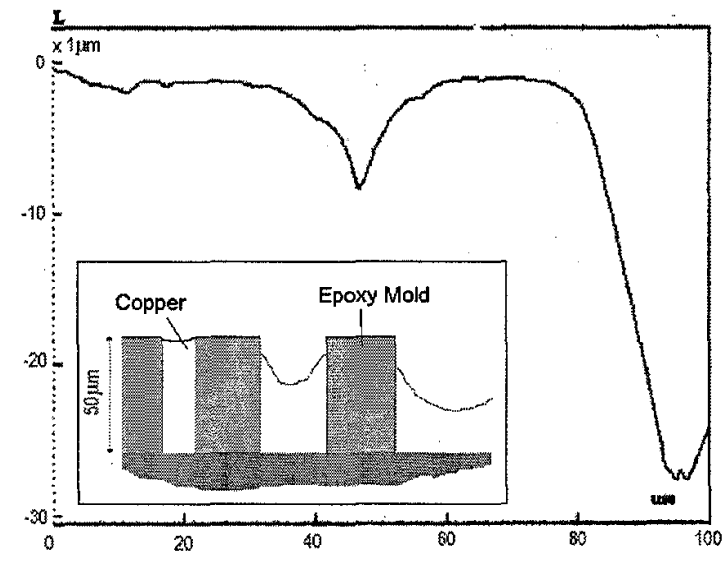

Figure 2.b: Profile of the filled trenches as measured using a surface profilometer. Although the trench depths measured in this manner are only semiquantitative, due to the finite curvature of the profilometer stylus, they corroborate the visual SEM image of filled trench trends shown in Figure 2.a. 
It was observed that when the copper was etched back and stopped prematurely, some residual copper was found accumulated at the bottom of the low aspect-ratio trenches. This was especially true at the corners where the sidewall meets the trench floor. This phenomenon can be attributed to the fact that the copper thickness is greater at the corners than at the planar surfaces.

For a $50 \mu \mathrm{m}$ thick mold, it was observed that in order to keep the copper intact in a trench with an aspect-ratio of 5, the lowest aspect-ratio that can be etched is 0.8 . In other words, simply as a function of dimensional differences, the ratio between the narrowest width to the widest width should be 1-to-5 for this mold thickness. This will allow for optimal utilization of the singlemask process.

Figures 3 and 4 show photomicrographs of functional electromagnets fabricated using this single-mask technique and the full process of Figure 1. The copper line width is $50 \mu \mathrm{m}$ and the initial height of the epoxy mold is also $50 \mu \mathrm{m}$. Pads for electrical contact were created by electroplating $\mathrm{Cu}$ around and in between an array of epoxy posts.

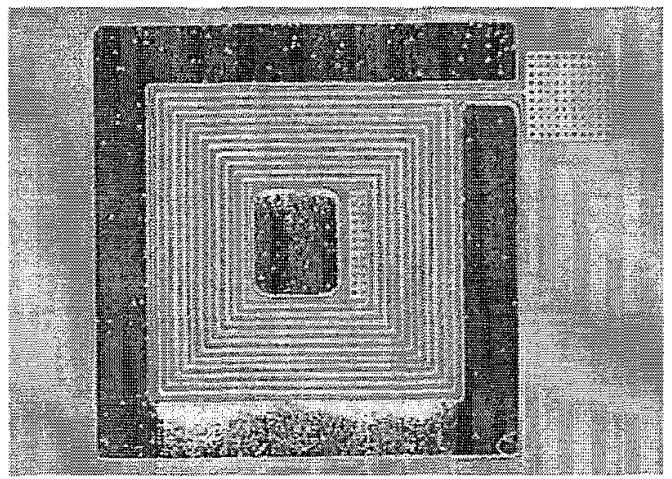

Figure 2: Photomicrograph of fabricated device with 50 um coil line width. Silver-colored areas represent NiFe magnetic cores.

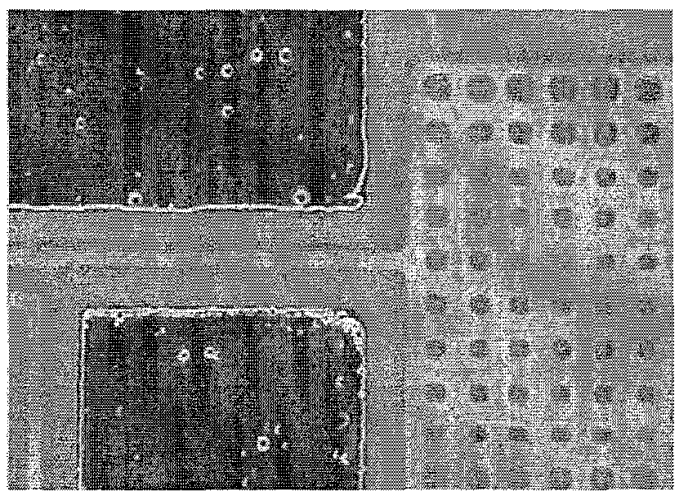

Figure 3: Close view of Fig 2.

Micro-coils with smaller line widths have also been successfully fabricated using the single-mask process. Figure 4 shows a detail of $15 \mu \mathrm{m}$ wide, clectrically isolated copper coils embedded in a $45 \mu \mathrm{m}$ thick epoxy mold. The upper surface of the copper lines has been etched back close to the surface of the epoxy. $\mathrm{NiFe}$ has been subsequently partially plated in the large opening. For maximum probability of successful fabrication, openings for permalloy material were at least an order of magnitude larger than the actual with of the copper wires.

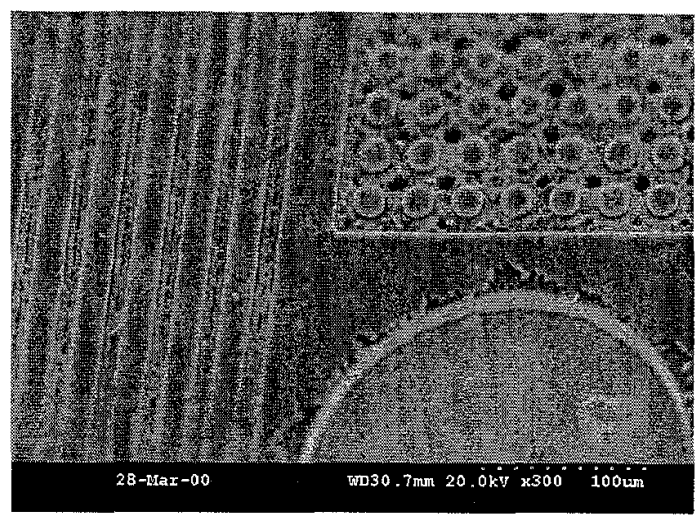

Figure 4: SEM micrograph of a micro-electromagnetic coil with $15 \mu \mathrm{m} \mathrm{Cu}$ coil linewidths. The semi-circle in the lower right of the micrograph is the tip of the partially filled NiFe magnetic core.

To demonstrate the functionality of these magnetic elements, measurement of the magnetic flux generated by these elements as a function of applied coil current were performed. The probe of a Bell 9550 Gauss/Teslameter was positioned over the central pole of the electromagnet of Figure 2 and the generated flux measured as a function of applied current. Figure 5 shows the dependence of generated magnetic flux as a function of applied coil current. Due to the large size of the gaussmeter probe relative to the coil, only a relative measurement of flux is possible. However, as expected, the current-flux relationship is linear, demonstrating proper operation of the electromagnet

\section{Current vs Magnetic Flux}

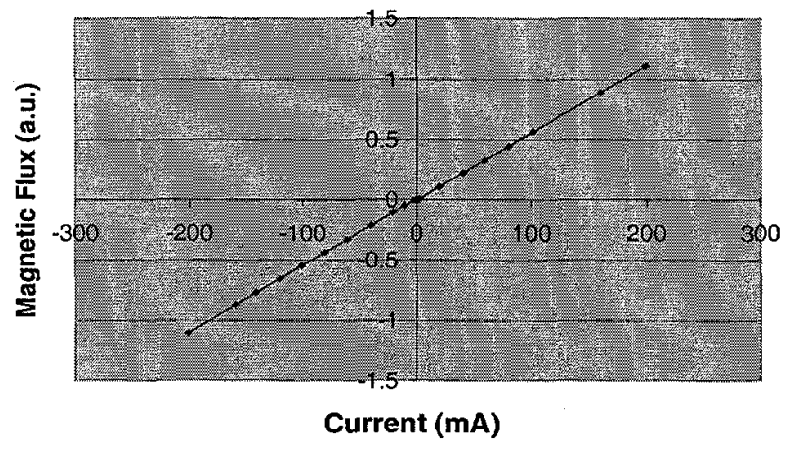

Figure 5: Flux generation as a function of current for the coil of Figure 2 .

\section{CONCLUSIONS}

A simplified methodology for the microfabrication of integrated electromagnetic structures using a self-aligned, singlemask process has been developed. It exploits differences in aspect-ratios for the differential filling of mold trenches using a combination of conformal and standard electrodeposition-throughmold techniques. The electroplating mold is retained after processing as a planarized surface for subsequent surface micromachining. Utilizing this technology, integrated micro-coils of 15 and $50 \mu \mathrm{m}$ have been fabricated and their magnetic performance measured. Although this process has been presented for magnetic micromachined devices incorporating windings and magnetic cores, it is also applicable to a wide variety of multimaterial structures and devices, as well as highly threedimensional structures such as inductors. 


\section{REFERENCES}

1. Park, J.Y., Bhattacharya, S.K., Allen, M.G., "Fully integrated passives modules for filter applications using low temperature processes", Proceedings. 1997 International Symposium on Microelectronics, Philadelphia, PA, USA, 14-16 Oct. 1997, SPIE vol.3235, p.592-7

2. Liakopoulos, T.M., Ming Xu; Chong Ahn, Nelson, T.L., "Ultrahigh resolution DC magnetic field measurements using microfabricated fluxgate sensor chips", Proceedings of Conference on Precision Electromagnetic: Measurements, 1998 Conference on Precision Electromagnetic Measurements Digest, Washington, DC, USA, 6-10 July 1998, p.630-1

3. Taylor, W.P., Brand, O., Allen, M.G., "Fully integrated magnetically actuated micromachined relays", Journal of Microelectromcchanical Systems, vol.7, no.2, June 1998, p.181-91

4. Epstein, A.H., Senturia, S.D., Anathasuresh, G., Ayon, A., Breuer, K., Chen, K.S., Ehrich, F., Gauba, G., Ghodssi, R., Groshenry, C., Jacobson, S., Lang, J., Mehra, C.C., Mur Miranda, J., Nagle, S., Orr, D., Piekos, E., Schmidt, M., Shirley, G., Spearing, S., Tan, C., Tzeng, Y.S., Waitz, I., "Power MEMS and microengines", Transducers 97. 1997 International Conference on Solid-State Sensors and Actuators: Digest of Technical Papers, Chicago, IL, USA, 16-19, vol.2, June 1997, p.753-6

5. Romankiw, L.T., "Evolution of the plating through lithographic mask technology", Proceedings of the Fourth International Symposium on Magnetic Materials, Processes, and Devices. Applications to Storage and Microelectromechanical Systems (MEMS), Chicago, IL, USA, 9-12 Oct. 1995, p.253-72

6. Carey, D.H., Pietila, D.A., Sigmond, D.M., "Trenching Techniques for Forming Channels, Vias, and Components in Substrates", U.S. Patent US05219787.

7. Richardson, D.L.K., Knowles, J.C., "Integral Honeycomb-like Support of Very Thin Single Crystal Slices", U.S. Patent US03936329.

8. Keller, C., Ferrari, M. "Milli-scale polysilicon structures", Technical Digest Solid-State Sensor and Actuator Workshop, Hilton Head Island, SC, USA, 13-16 June 1994, p.132-7

9. Conedera, V., Le Goff, B., Fabre, N., "Potentialities of a new positive photoresist for the realization of thick moulds", Journal of Micromechanics and Microengineering vol.9, no.2, June 1999, p.173-5

10. Lorenz, H., Despont, M., Fahrni, N., Brugger, J., Vettiger, P., Renaud, P., "High-aspect-ratio, ultrathick, negative-tone near-UV photoresist and its applications for MEMS", ELE Tenth Annual International Workshop on Micro Electro Mechanical Systems. An Investigation of Micro Structures, Sensors, Actuators, Machines and Robots, Nagoya, Japan, 26-30 Jan. 1997, vol.A64, no.1, p.33-9

11. Chang H.K., Kim Y.K., "UV-LIGA process for high aspect ratio structures using stress barrier and Cshaped etch holes", Transducers 99, June 7-10, Sendai, Japan, vol.2, p1428-1431

12. Lorenz, H., Despont, M., Fahrnl, N., LaBianca, N., Renaud, P., Vettiger, P., "SU-8: a low-cost negative resist for MEMS", Journal of Micromechanics and Microengineering, vol.7, no.3, p.121-4 